NASA Technical Memorandum 85686

NASA-TM-8568619840007111

CAPTURE-EJECTOR SATELLITES

Ian O. MacConochie,

Charles H. Eldred,

and James A. Martin

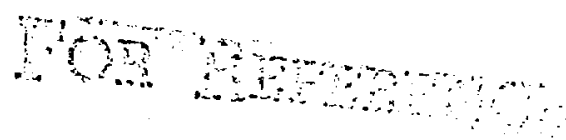

\title{
October 1983
}

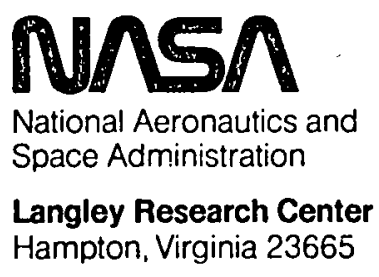

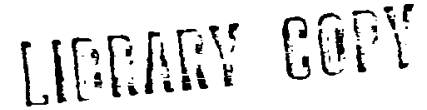

\{:ic 1333

WNGLEY FESEARCH CENTER LIERARY, MASA

HA:TTOL VIRGINIA 
-

. 


\title{
CAPTURE-EJECTOR SATELLITES
}

\author{
by Ian 0. MacConochie, \\ Charles H. Eldred, \\ and James A. Martin \\ Langley Research Center, Hampton, VA \\ SUMMARY
}

In this paper, a satellite in the form of a large rotating rim is described which can be used to boost payloads from low-Earth orbit to higher orbits. The rim rotates in the plane of its orbit such that the lower portion of the rim is traveling at less than orbital velocity, while the upper portion is traveling at greater than orbital velocity. The ascending transport arrives at the lowest portion of the rim at less than orbital velocities and discharges its payload for attachment to the rim's perimeter. The payload remains on that portion of the rim until it reaches its highest point where it is released on a trajectory for higher orbits. Transfers from a high to a low-Earth orbit, or entry, are accomplished by employing the reverse procedure. When using the Capture-Ejector Rim System on orbit, significant reductions in the size and number of flights for the delivery transport are possible.

\section{INTRODUCTION}

Several proposals have been made for large satellites or towers which could serve as gravity ladders. ${ }^{1-3}$ Their purpose is to reduce energy requirements for spacecraft traveling between the Earth and various orbits. In one proposal, a geostationary tower would be constructed to extend from the Earth's surface to $81,000 \mathrm{n}$. mi. altitude or well beyond the altitude required for geosynchronous orbit (GE0). GEO satellites could 
be launched from Earth by ascending the tower to the required altitude. Also, by allowing the rotation of the tower to accelerate payloads upward from CEO altitude, the tower's rotational energy could be used to launch probes without rockets to the Moon or planets. ${ }^{1}$ In another proposal, a large satellite consisting of two long in-line spokes would act like a giant wheel rolling around the Earth. ${ }^{3}$ The satellite, 2,500 miles long, would touch the Earth's surface twice every revolution, each spoke depositing or picking up payloads. Atmospheric velocities up to $6,000 \mathrm{ft} / \mathrm{sec}$ are generated, however, creating aerothermodynamic issues related to dynamic pressure, drag, heating, and sonic boom.

\section{SYMBOLS}

$$
\begin{aligned}
& \mathrm{a}=\text { centrifugal acceleration, } \mathrm{ft} / \mathrm{sec}^{2} \\
& A=r i m \text { cross-sectional area, } \mathrm{ft}^{2} \\
& \mathrm{~g}=\text { gravitational constant, } 32.2 \mathrm{ft} / \mathrm{sec}^{2} \\
& n=\text { multiples of } \mathrm{g} \\
& r \text { = radius of } \mathrm{rim}, \mathrm{ft} \\
& V=\mathrm{rim} \text { velocity, } \mathrm{ft} / \mathrm{sec} \\
& V_{\text {max }}=\text { maximum rim velocity, } f t / s e c \\
& W_{P}=\text { weight of payload, Ib } \\
& W_{r}=\text { welght of rim, } 1 b \\
& \varepsilon \quad=\text { eccentricity } \\
& \rho=\text { material weight density, } 1 b / f t^{3} \\
& \sigma=\text { stress, } 1 b / f^{2}
\end{aligned}
$$




\section{DESCRIPTION OF THE CAPTURE-EJECTOR RIM}

Instead of permanent towers or rotating spokes, the authors propose a plain rim (Figs. 1 and 2), referred to herein as a Capture-Ejector Rim. An advantage of the rim over a rotating tower (or spokes) is the relative ease with which phasing for landing or takeoff can be accomplished. However, like the satellite with two spokes, the rim rotates about its own axis in the same direction as the orbital motion, traveling at some preselected altitude--an altitude which represents a compromise between station-keeping propellant to overcome drag and basic mission requirements. In this design, each spacecraft arrives at suborbital velocity and attaches to the lower portion of the rim and is released from the rim at its high point at super orbital velocity. For return to Earth, the reverse procedure is used; that is, returning spacecraft attach to the top of the rim and ride the rim to the low point where the spacecraft are ejected at suborbital velocity for entry into the Earth's atmosphere.

Because of conservation of energy, the rim will slow down if the mass of all outgoing spacecraft exceeds the mass of all returning spacecraft. Mission models for traffic to and from Earth suggest that the summation of the masses of propellants and spacecraft leaving will exceed that returning by a ratio of approximately 4 to 1 . Therefore, some type of propulsion system on the rim is needed to restore energy. This added impulse might be derived from solar-powered ion thrusters. ${ }^{4}$ These engines use electrical power from the Sun to ionize a gas which produces thrust at a specific impulse of 2,000 to 10,000 seconds compared to chemical rocket engines which produce thrust at a maximum specific impulse of less than 500 
seconds. Herein lies one of the advantages of the Capture-Ejector Rim, since highly efficient engines, producing low thrust over long periods of time, can build up rotational energy in the rim which can be stored for use at very rapid rates when required.

The baseline design for the Capture-Ejector Rim consists of a simple rectangular rim cross section. No spokes or tension ties are needed. In Fig. 2, a spacecraft is shown landing inside the rim. A more probable design, however, might involve an arresting system wherein the payload delivered to low-Earth orbit is suspended from the outside of the rim as shown in Fig. 3. In order to attach to the rim, a yoke with a scissors arrangement is used. Tires are mounted in the arresting gear in order to permit some relative tangential and radial velocity at contact between the arresting gear and rim. Additional terminal radial velocity is also permissible with the use of shock absorbers mounted in the yoke of the grappling device.

Ion thrusters are mounted on the exterior of the rim with thrust axes parallel to the rim circumference. Argon is selected as the propellant to minimize potential atmospheric contamination. The engines provide the necessary impulse to restore kinetic energy to the system after payload capture and ejection and to provide the necessary station-keeping impulse. To perform the station-keeping function, the engines (normally operated in diametrically opposite pairs) are operated singly to give the rim a posigrade impulse.

RIM VELOCITY, SIZING, AND DESIGN CONSTRAINTS

Several factors will govern the rim velocity and physical size. Rim velocity, for example, will be governed by the intended mission in 
conjunction with available materials; rim radius by the established acceleration limits for the selected velocity; and total rim mass by the mass of the payloads to be transferred and rim rotational eccentricity allowed.

For a simple rim freely rotating in space with no payload, the maximum allowable rim speed is only governed by the specific strength of the rim material and is independent of rim radius or:

$$
v_{\max }=\sqrt{\frac{\sigma g}{\rho}}
$$

This relationship can be derived by considering a segment of the rim with inertial acceleration balanced by the components of the tensile stress in the rim. (See Appendix A.) For purposes of illustration, assume a composite material is used having an ultimate tensile strength of $27.7 \times 10^{6}$ $\mathrm{lb} / \mathrm{ft}^{2}$ and a density of $95 \mathrm{lb} / \mathrm{ft}^{3}$; the specific strength of this material is $9.39 \times 10^{6} \mathrm{ft}^{2} / \mathrm{sec}^{2}$, giving a maximum achievable speed based on Eq. (1) of $3060 \mathrm{ft} / \mathrm{sec}$. This achievable rim speed versus specific strength is shown on Fig. 4. The approximate limit for current materials is shown at point 1 , and the required specific strength for placing a spacecraft on a trajectory which would take it to GEO is shown at point 2 . Of course, the actual rim velocity achievable would be less than the theoretical because of the dynamic loads imposed on the rim by arriving and departing spacecraft. These added loads would tend to raise the stress in the rim above that caused just by rotation.

As noted from Eq. (1), the velocity of the Capture-Ejector Rim can be run is independent of the centrifugal acceleration. For practical reasons, however, acceleration levels are limited at the rim. For humans, an 
acceleration of $1 \mathrm{~g}$ would certainly be acceptable. With increasing acceleration levels, maneuvering becomes increasingly difficult (for example, for astronauts to operate controls). The current Space Shuttle during ascent has been designed for a peak acceleration of $3 \mathrm{~g}$ 's. For unmanned transfers of equipment, spacecraft, or supplies much higher $g$ levels may be acceptable. To find the smallest rim acceptable for the current system (a desirable goal from a rim cost standpoint), rim velocity and permissible acceleration must be considered, or:

$$
r=\frac{v^{2}}{n g}
$$

As evident from Eq. (2) and as shown in Fig. 5, the utilization of higher acceleration results in substantial reductions in rim radii for the same required transfer velocities. For a 3,000 ft/sec $\mathrm{rim}$ speed requirement, for example, rim radius is reduced from $280,000 \mathrm{ft}(46 \mathrm{n}$. mi.) to $28,000 \mathrm{ft}(4.6 \mathrm{n} . \mathrm{mi}$.$) by increasing design acceleration from 1 \mathrm{~g}$ to $10 \mathrm{~g}$.

\section{RIM SIZING}

In Fig. 4, rim velocities in terms of stress-to-density ratios of materials were plotted using Eq. (1). In actual practice, the required mission velocity is the primary consideration dictating stress in the rim (Fig. 6). As shown from Eq. (1), once a velocity requirement and the material density are identified, stress in the rim is established. The design condition for a rim cross section is assumed to be based on the acceptable eccentricity for the rim when the payload is in place. This eccentricity is found simply by taking the ratio of the mass of the payload being transferred divided by the weight of both payload and rim, or: 


$$
\varepsilon=\frac{W_{p}}{W_{p}+W_{r}}
$$

Solving for rim weight:

$$
W_{r}=\frac{(1-\varepsilon) W_{p}}{\varepsilon}
$$

Rim weight from Eq. 4 versus eccentricity is plotted in Fig. 7 for three different payloads. Obviously, the larger the payload weight, the larger the rim weight needed to minimize the eccentricity. As an illustrative example, assume that the permissible eccentricity is 0.02 for a payload of $65,000 \mathrm{lb}$, then the required $\mathrm{rim}$ weight is $3.2 \times 10^{6} \mathrm{lb}$ (e.g. design baseline in Fig. 7). If, on the other hand, the payload to be transferred is only $10,000 \mathrm{lb}$, then the rim weight required is only $492,000 \mathrm{lb}$ for the same eccentricity.

In the design process then, first the boost velocity requirement for the mission is established; secondly, a material is selected having adequate strength-to-density ratio such that the required rim velocity is less than $V_{\max }$ given by $\mathrm{Eq} .(1)$; thirdly, a rim radius is calculated from Eq. (2) which complies with the specified limits on centrifugal acceleration for the payload on the rim; and lastly, an eccentricity limit is established. This last requirement establishes rim mass and, therefore, the rim cross-sectional area to be used for the radius and rim velocity selected.

Because Capture-Ejector Rims tend to be so massive, tradeoffs would have to be made to determine benefits based on anticipated traffic versus 
the cost of delivery to orbit of the necessary materials to build the device. For example, at 65,000 lb per Shuttle flight, approximately 50 Shuttle flights would be required for the $3.2 \times 10^{6}$ lb baseline rim cited in Fig. 7. One way to reduce these flights would be to drastically reduce the payload allowed on the rim with greatly increased rim utilization for delivery of the same total payload weight to orbit. For example, a rim, limited in payload to $10,000 \mathrm{lb}$ and an eccentricity of 0.02 , would weigh 492,000 $\mathrm{lb}$ and would require only eight Shuttle flights for delivery of the basic structure. One problem, however, would entail the logistics involved in the delivery of payloads in 10,000 $\mathrm{lb}$ increments when the basic vehicle is capable of delivering payloads in 65,000 lb increments.

\section{LOW-EARTH ORBIT TO A SPACE STATION}

An Earth orbital application is one of the most useful for the CaptureEjector Rim. For this application, the device could be placed in low-Earth orbit and used to boost payloads to higher orbits; for example, to about $270 \mathrm{n}$. mi., a possible space station orbital altitude range. Assume that the Capture-Ejector Rim is placed in an orbit so that the lowest portion extends down to a $6 \times 10^{6} \mathrm{ft}(100 \mathrm{n}$. mi.) altitude. In order to eject a spacecraft onto a trajectory whose apogee reaches a suitable altitude for a space station, a rim velocity of about $300 \mathrm{ft} / \mathrm{sec}$ is required.

For this application assume that eccentricity of the rotating rim is to be limited to 0.02 . Further assume that the same composite referred to earlier is used having a rim velocity limit of $3,060 \mathrm{ft} / \mathrm{sec}$. Since the required rim velocity for the current mission is only $300 \mathrm{ft} / \mathrm{sec}$, the material selected is more than adequate. From Eq. (2) and assuming a $1 \mathrm{~g}$ 
limit for centrifugal acceleration for payloads on the $r i m$, the required radius is $2,800 \mathrm{ft}$. From Eq. (4) the required mass is $3.2 \times 10^{6} \mathrm{lb}$.

The relationship between rim material density, cross sectional area, radius, and rim mass is given by:

$$
W_{r}=2 \pi \rho A r
$$

Then the required area is $1.9 \mathrm{ft}^{2}$ for the $95 \mathrm{lb} / \mathrm{ft}^{3}$ material and $2,800 \mathrm{ft}$ radius. A rim 3 inches thick by 92 inches wide provides the required area. The thickness will provide some resistance to flexure, and the width provides space for solar panels and ion engine installations on the outside perimeter of the rim.

Dynamic loads during attachment of payloads and the inertial load of subsystems will raise rim stress. Design margins are still more than adequate, since the operating stress levels for the space station mission, even when assuming a dynamic factor of 3 for landing and 0.1 for inertial load of subsystems, will result in a limit operating rim stress of only an estimated 2.8 percent of material ultimate. As stated earlier, the large weight of the rim is not derived from stress considerations in this analysis, but is based on eccentricity of motion permissible when payloads are attached.

The rim will lose 4.5 percent of its rim velocity and 8.8 percent of stored kinetic energy from the capture and ejection of a single $65,000 \mathrm{lb}$ payload. This loss can be restored using 120 ion thrusters with a thrust of $0.0225 \mathrm{lb}$ each equally spaced around the perimeter of the rim and operating at an assumed 3000 seconds specific impulse for 5.7 days. The propellant consumed is $445 \mathrm{lb}$ over this period. At apogee, a circularization 
burn is needed for the released spacecraft, but a substantial savings is still realized by virtue of elimination of the perigee burn on a tug which would otherwise be required.

The reduction in the required launch vehicle energy can be realized either as an increased payload for a given launch vehicle (reduced number of flights for a given mission model) or as a reduction in the required launch vehicle size for a given payload. The potential savings in launch vehicle weight as the result of size reduction is shown in Fig. 8 and is based on differences between the use of a Capture-Ejector boost from $6 x$ $10^{6} \mathrm{ft}$ (100 n.mi.) altitude in confunction with the Earth-to-orbit delivery vehicle and a mission wherein the launch vehicle discharges the payload directly at apogee from an orbit whose perigee is $100 \mathrm{n}$. mi. and whose apogee is at space station altitude.

When viewed in this light, the savings in launch vehicle gross weight is $500,000 \mathrm{lb}$ for a required delivery of $65,000 \mathrm{lb}$ to a space station orbit i.e. the vertical distance between the solid lines at a relative velocity of 25,900 ft/sec in Fig. 8. Again, the savings in launch vehicle weight is due to the suborbital velocity of the Capture-Ejector Rim at $100 \mathrm{n}$. mi. altitude permitting the undersizing of the launch vehicle for delivery of the payloads at less than orbital velocity; this savings is coupled with the savings from the posigrade boost given the payload by the rim at the ejection point which carries the payload to the space station orbit.

When operating a Capture-Ejector Rim at its material limit of 3,060 $\mathrm{ft} / \mathrm{sec}(28,500 \mathrm{ft} / \mathrm{sec}$ relative velocity in $\mathrm{Fig} .8)$, a savings of over 40 percent in launch vehicle weight alone is achieved by virtue of the suborbital motion of the system. (The estimates are based on methods used in 
reference 5.) The savings are represented in Fig. 8 as a decrease in Earth-to-orbit (ETO) transport weight (lower curve in the figure). The upper curve in the figure represents the weight of the ETO required if no Capture-Ejector Rim (CER) is used to develop the relative velocity shown. High values of perigee velocity such as $28,500 \mathrm{ft} / \mathrm{sec}$ and $10,000,000$ Ib launch vehicle weight are, of course, not realistic, and direct delivery at this energy level without the use of a space tug (or a Capture Ejector Rim) does not represent a realistic mission. The curve, however, is shown for comparison purposes.

Fuel consumption required for the ion engines on the rim is approximately one seventh that required for an equal thrust chemical engine. Although argon has been considered as the fuel to drive the rim, the utilization of residual and unused reserve propellants from arriving and departing spacecraft may be possible and advantageous. In any event, propellant must be obtained in this manner or charged against the system weight savings just identified in Fig. 8.

\section{LEO TO GEO}

A mission involving transit to GEO is another potential application for a Capture-Ejector Rim, since traffic to this orbit is large and is expected to grow. In order to eject a payload into such an orbit with an apogee at geosynchronous altitude, a rim speed of approximately $7,500 \mathrm{ft} / \mathrm{sec}$ is needed. The required specific strength of a material for this application is $56.3 \times 10^{6} \mathrm{ft}^{2} / \mathrm{sec}^{2}$ (Point 2 in Fig. 4) or about six times the specific strength of any currently available material. For an assumed 3-g limit and a $7,500 \mathrm{ft} / \mathrm{sec}$ rim velocity, the required rim radius is $5.8 \times 10^{5} \mathrm{ft}$. 
Applying the same ground rules to this system, namely an eccentricity limit of 0.02 for a 65,000 lb payload, the cross-sectional area of the rim will be $0.00919 \mathrm{ft}^{2}\left(1.32 \mathrm{in}^{2}\right)$. A belt 0.1 inches thick by 13.2 inches wide could be used. Because of the space required to mount ion engines and lack of rigidity, it may be necessary to increase rim size beyond that required just for minimization of eccentricity.

The rim's mass center is located at a $11.9 \times 10^{5} \mathrm{ft}$ altitude so that the lowest portion of the rim extends down to $6.1 \times 10^{5} \mathrm{ft}(100 \mathrm{n}$. mi.) altitude. Because of the suborbital velocity of the rim, the Earth-to-orbit transport can be reduced substantially in weight as determined by the same methods used in Ref. 5. In addition, more of the 65,000 lb cargo delivered to LEO can be assigned to payload for the GEO destination, since the transferring space tug can be reduced in size because of the perigee impulse from the Capture-Ejector Rim. As a final result, an estimated two out of every five Earth-to-LEO trips could be eliminated in addition to downsizing the Earth-to-LEO vehicle.

PARTIAL $\triangle V^{\prime}$ 'S AND MULTIPLE RIMS

Partial $\Delta V^{\prime}$ 's may be useful for GEO and many other missions. What the Capture-Ejector Rim does not provide, a chemical rocket motor could. Since the ejected spacecraft must have a rocket engine and tankage for circularization, providing partial boosts using a Capture-Ejector system means that less impulse is required from the chemical propulsion system.

In another mission, a Capture-Ejector Rim stationed at LEO provides the partial perigee boost for a GEO destination. This is followed immediately by a boost from the chemical propulsion system adequate for a Hohmann transfer to GEO. At GEO, the chemical propulsion system is again used, this time for a circularization burn. 
Applying the same ground rules to this system, namely an eccentricity limit of 0.02 for a 65,000 lb payload, the cross-sectional area of the rim rims are at different altitudes and have different periods, it is only necessary to wait until the transfer opportunities present themselves. Precise timing and mid-course corrections are required. It would be necessary for the captured spacecraft on the lower rim to drive itself around the rim so that the spacecraft is in the proper location on the rim for the release. (Note: A spacecraft on wheels driven inside the rim will exchange momentum with the rim when the payload is moving counter to the motion of the rim in an amount inversely proportional to weight.) The precision and programing of such a series of maneuvers would be complex; however, it should be remembered that release from a rotating rim in space should be quick, easy, and precise.

\section{MANUFACTURE OF SATELLITES}

The Capture-Ejector Rims could be manufactured in orbit using a pultrusion process. 6 Using this process, a continuous structural section can be manufactured, the only limitation being the supply of fiber reinforcement and matrix material. A system for manufacturing the satellite using the Space Shuttle as a flatbed is shown in Fig. 10. In this arrangement, the pultrusion machine $(A)$ is installed so that the pultruded material travels perpendicular to the principal axis of the vehicle. Because curvature is so small for large satellites (and diameters, therefore, very large) some instrumentation is needed to continuously monitor the position of the extruded material in space relative to some reference. If the discharged composite material deviates from a circle of the required radius, a self-pulsed ranging laser described in Ref. 7 would send the information back in a servo-loop to alter the curvature of the pultruder dies as 
needed. Additionally, information on position of the extruded material during manufacture could be provided by utilizing a NASA developed infrared emitting diode and optical receiver system. This system, described in reference 8 , is capable of determining an exact position in an $x y$-plane normal to the diode beam. The latter system would be necessary if the pultruded material would not adhere to a perfect plane (normal to the curvature) over long lengths during the pultrusion process. Experimental work on pultrusion of various shapes on a pilot basis is in progress at the Langley Research Center. A pultrusion machine installed in the Shuttle is described in reference 9 for making a large truss structure on orbit--a proposal not too different from the current one except for the structural shape to be manufactured.

\section{SUMMARY REMARKS}

All of the gravity ladder concepts represent ambitious projects in terms of currently known technology and resources of any one nation or even groups of nations. From the standpoint of currently known materials, Capture-Ejector Rim speeds of approximately 3,000 ft/sec are theoretically possible; but to operate at this speed, the radius of the rim must be over $9.3 \times 10^{4} \mathrm{ft}(17 \mathrm{miles})$ in order to limit centrifugal acceleration to 3 g's. For transfer to GE0, a velocity increment of approximately 7,500 $\mathrm{ft} / \mathrm{sec}$ is required--a rim speed which far exceeds capabilities of currently known materials. Rim sizes for a $3 \mathrm{~g}$ limit are correspondingly higher. The Capture-Ejector concept may be very useful, however, for relatively small velocity increments such as supplying the boost needed to transfer from a low-Earth orbit to a space station. 


\section{APPENDIX A}

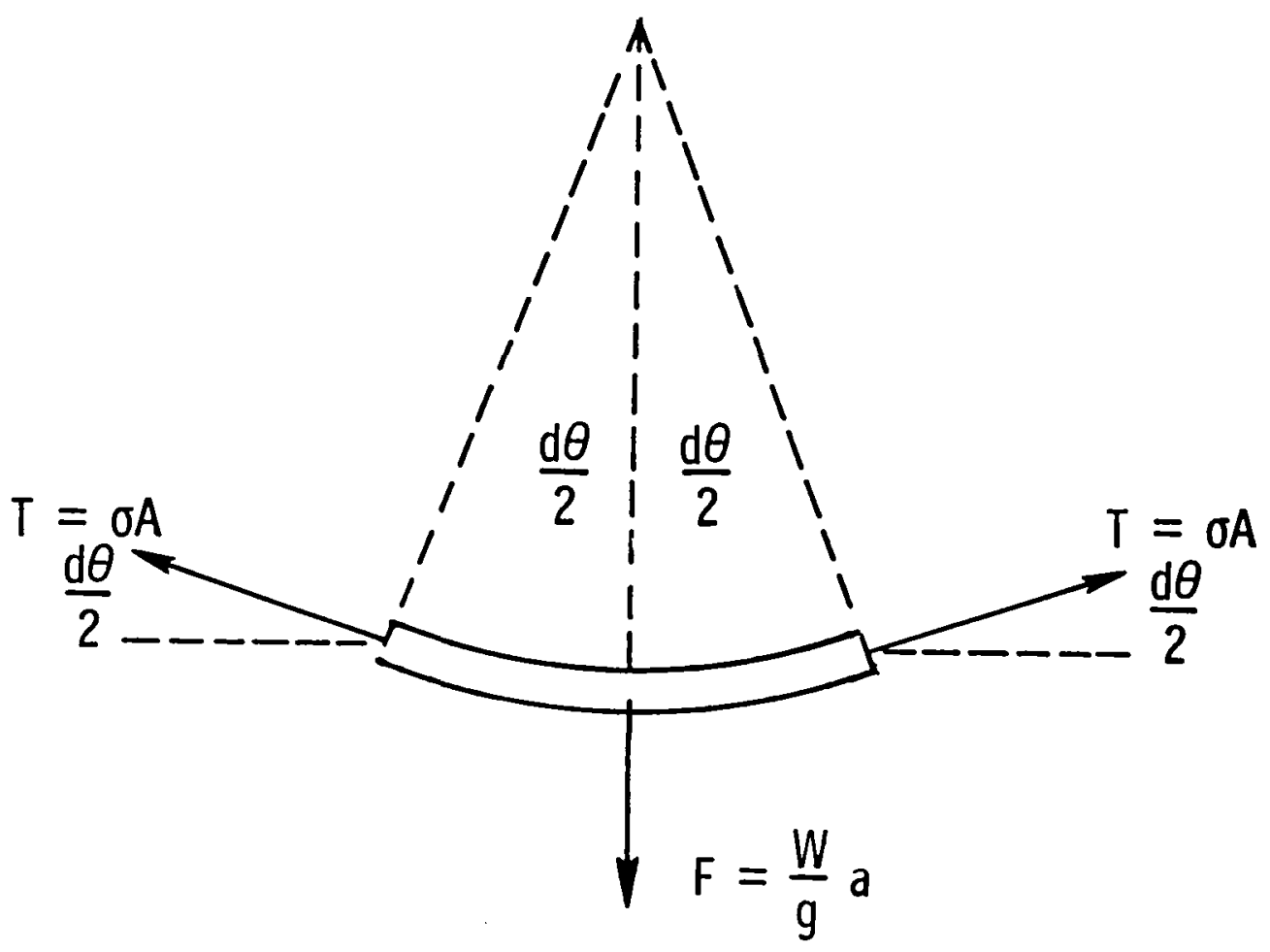

Summing forces in the radial direction for a rim element:

$$
\sum F=\frac{W}{g} a-2 \sigma A \sin \frac{d \theta}{2}=0
$$

Substituting for $W$, the product of rim density times volume of the segment, and replacing $\sin \frac{d \theta}{2}$ with $d \theta$ for small angles and $\frac{v^{2}}{r}$ for centrifugal acceleration, $a$, the above becomes:

$$
\frac{\rho \operatorname{Ard} \theta}{g} \quad \frac{v^{2}}{r}-2 \sigma A \frac{d \theta}{2}=0
$$

Canceling terms and solving for rim velocity:

$$
v=\sqrt{\frac{\sigma g}{\rho}}
$$




\section{REFERENCES}

1Pearson, J., "The Orbital Tower: A Spacecraft Launcher Using the Earth's Rotational Energy," Acta Astronautica, Vol. 2, p. 785, 1975.

${ }^{2}$ Pearson, J., "Anchored Lunar Satellites for Cislunar Transportation and Communication," Journal of Astronautical Sciences, Vol. XXVII, No. 1, pp. 39-62, January-March 1979.

${ }^{3}$ Moravec, Hans, "A Non-Synchronous Orbital Skyhook," Journal of Astronautical Sciences, Vol. XXV, No. 4, October-December 1977.

${ }^{4}$ Brewer, G. R., "Ion Propulsion Technology and Applications," Gordon and Breach - Science Publishers, New York, N.Y., 1970.

${ }^{5}$ Martin, James A., "Comparisons of Advanced Hydrocarbon Rocket Engines for Earth-to-orbit Vehicles," Journal of Spacecraft and Rockets, Vol. 20, No. 3, May-June 1983, pp. 249-256.

${ }^{6}$ Thompson, V. and Bradley, R. J., "Pultrusion of Advanced Composites," Soclety of Manufacturing Engineers, SME Paper No. EM76-415, 1976.

${ }^{7}$ Berdahl, M., "JPL Self-Pulsed Laser Surface Measurement System Development," NASA CP-2118, pp. 77-94, 1979.

${ }^{8}$ Spiers, R. B., Jr, "Surface Accuracy Measurements Sensor For Deployable Reflector Antennas," presented at the Large Space Systems Technology Conference-1980, NASA Conference Publication 2168, Vol. I - Systems Technology, pp. 439-448, November 18-20, 1980.

${ }^{9}$ Delmonte, J., "Technology of Carbon and Graphite Fiber Composites," Van Nostrand Reinhold Publishing Co., 1981. 


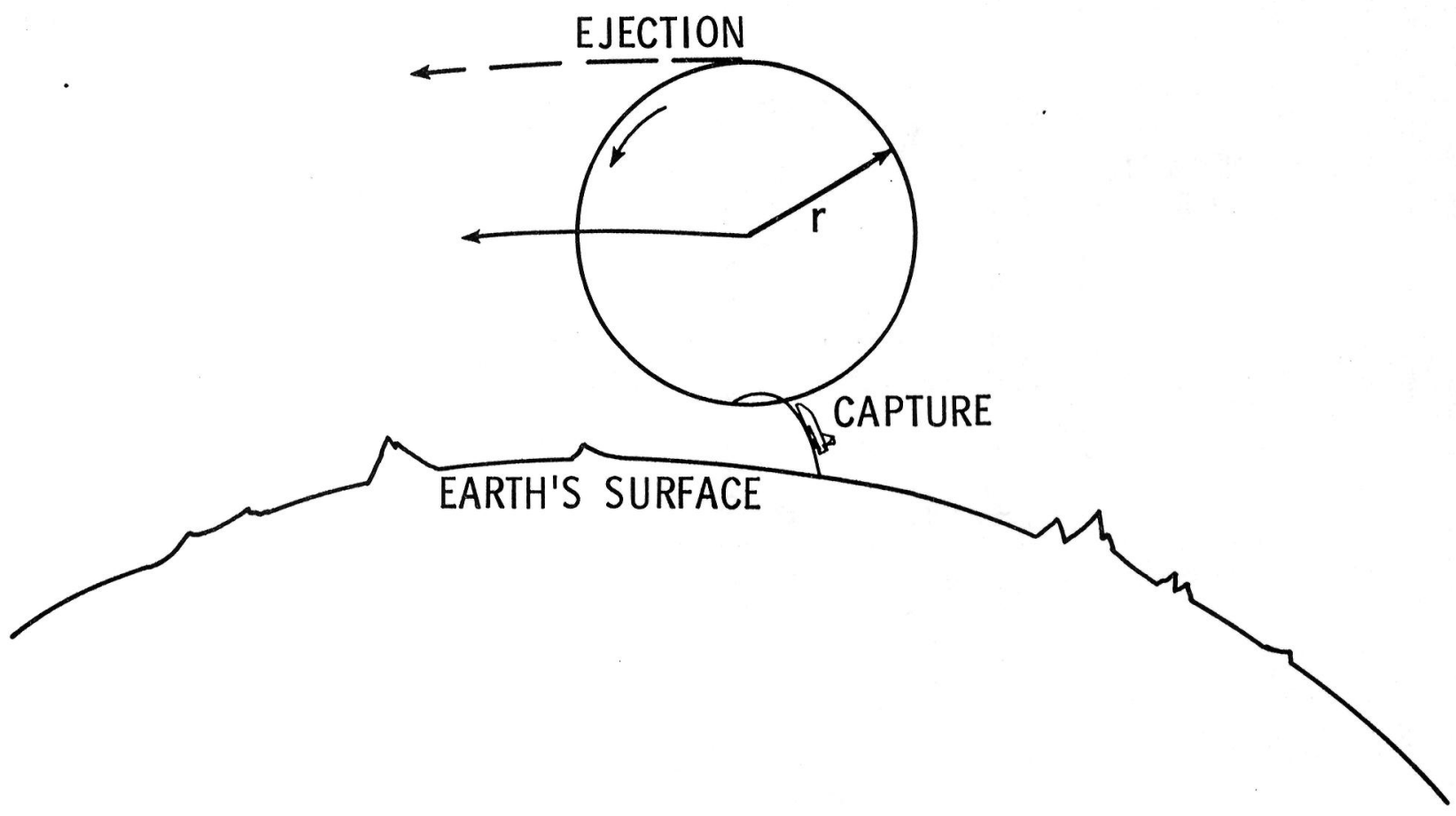

Fig. 1 The Capture-Ejector Satellite mission.

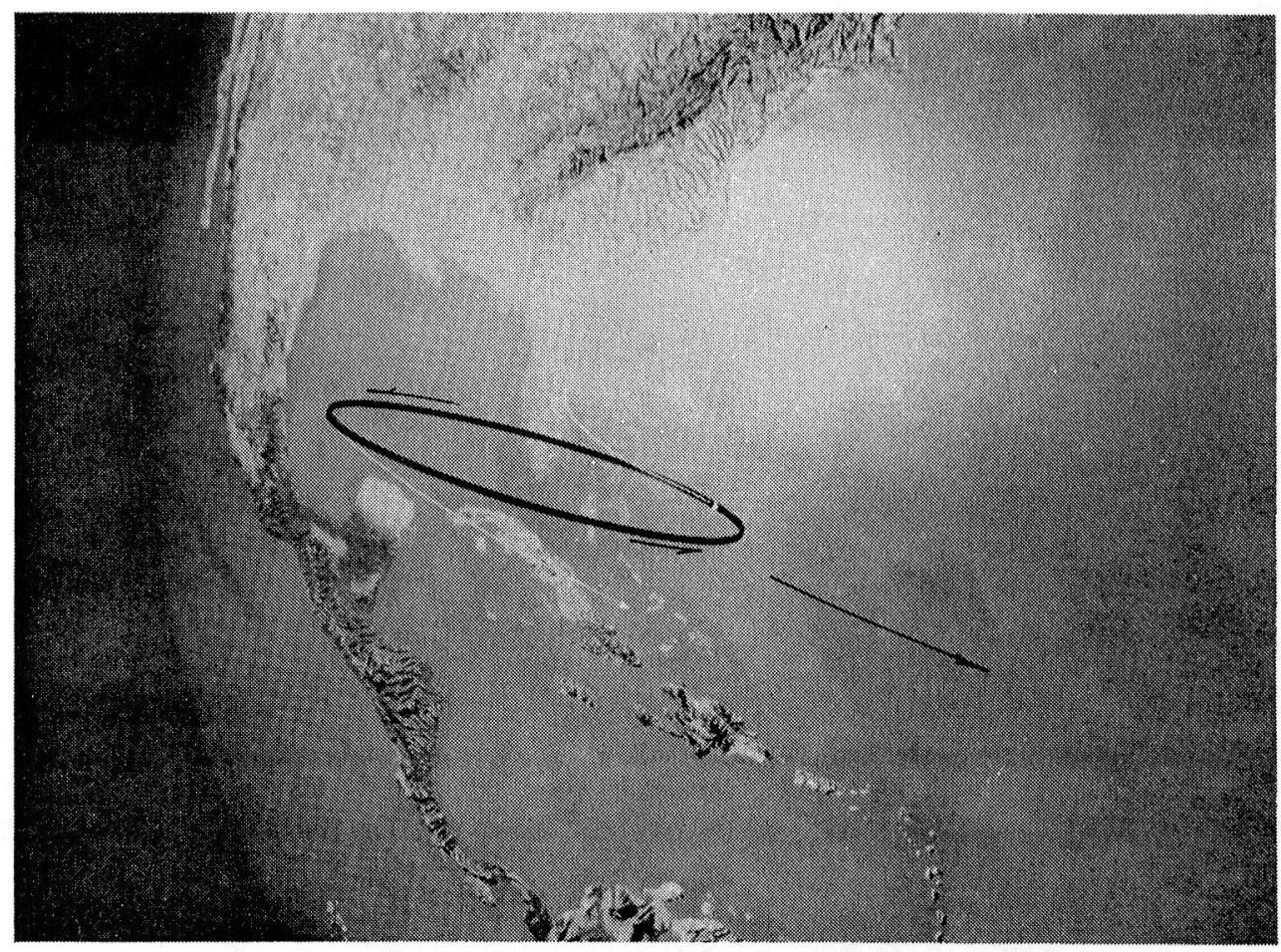

Fig. 2 Artist's concept of a Capture-Ejector Satellite. 


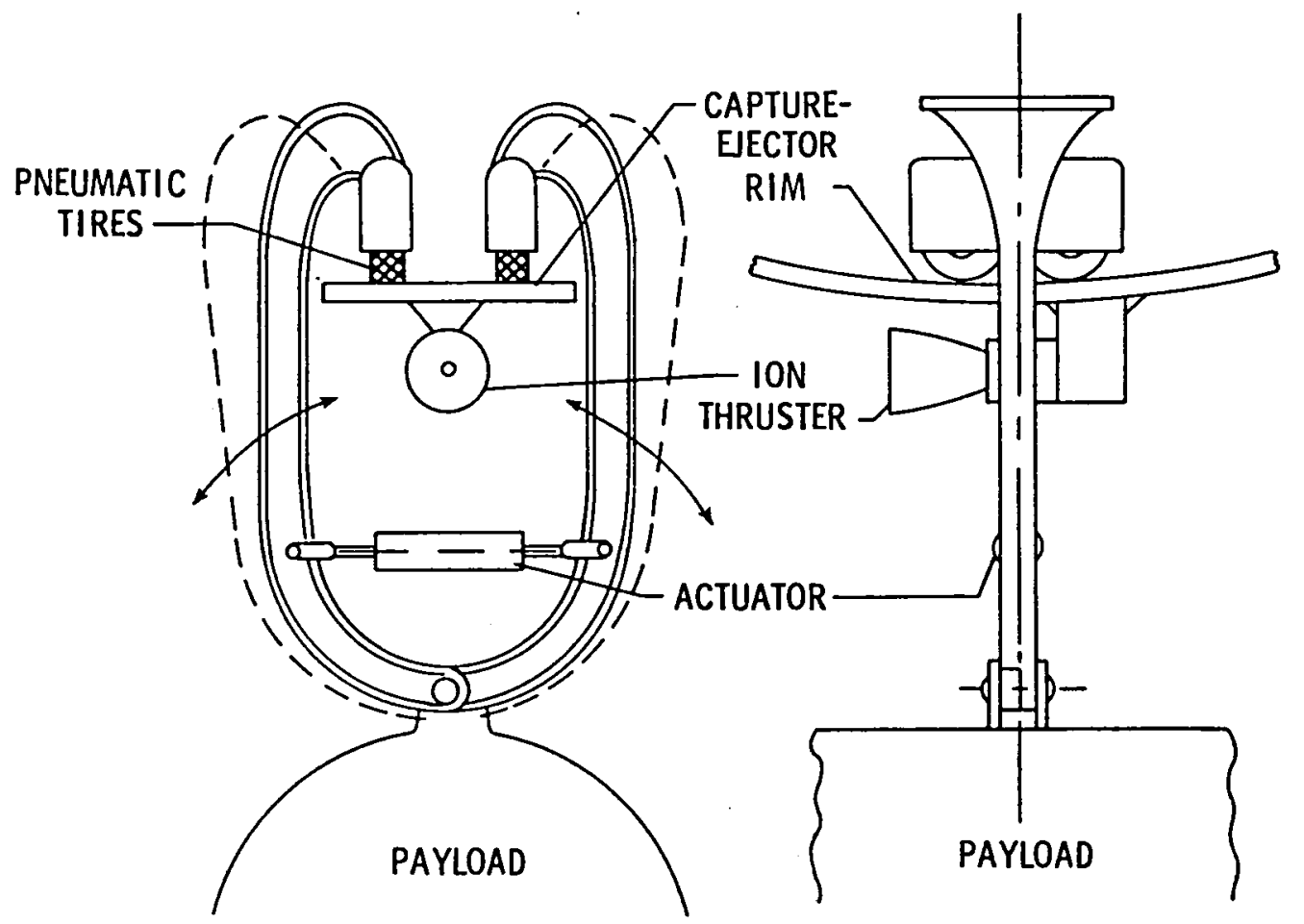

Fig. 3 Arresting gear for payload capture.

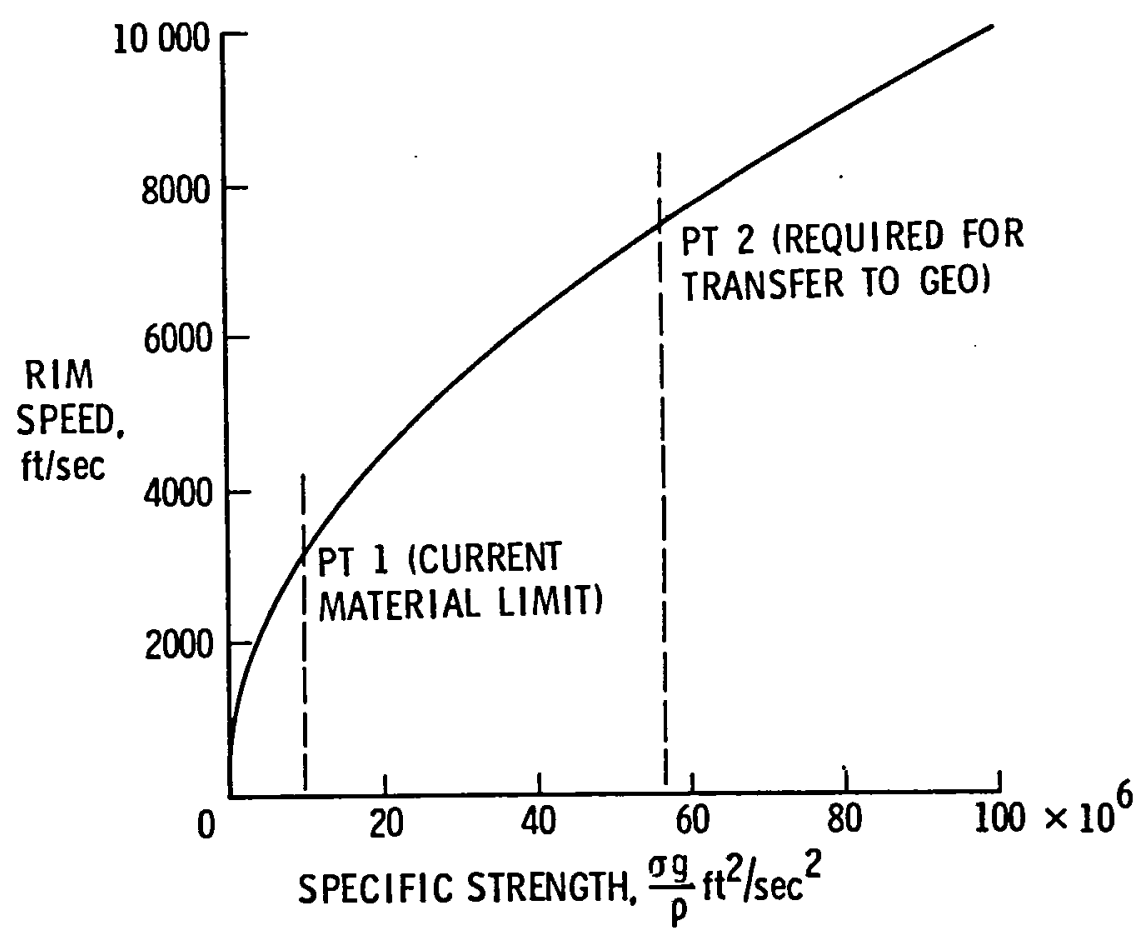

Fig. 4 Rim speed versus specific strength of rim material. 


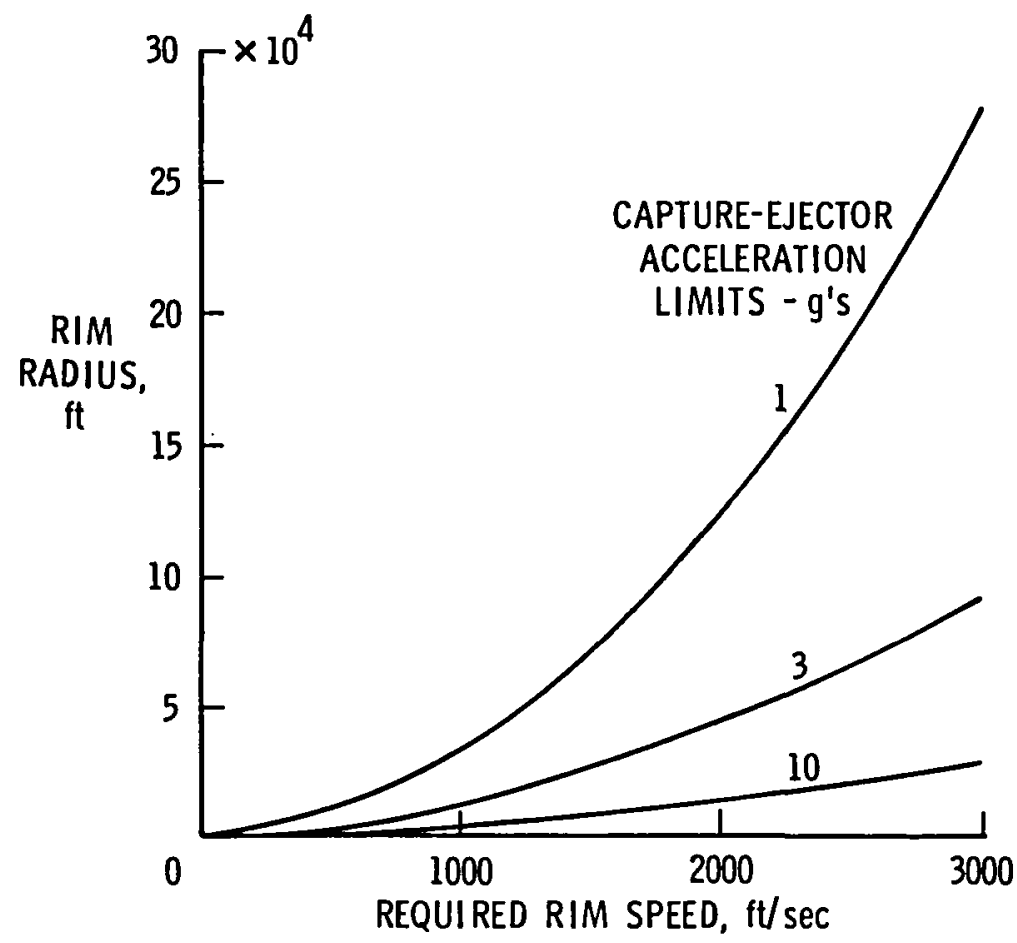

Fig. 5 Capture-Ejector Rim radius versus velocity for specified g limits.

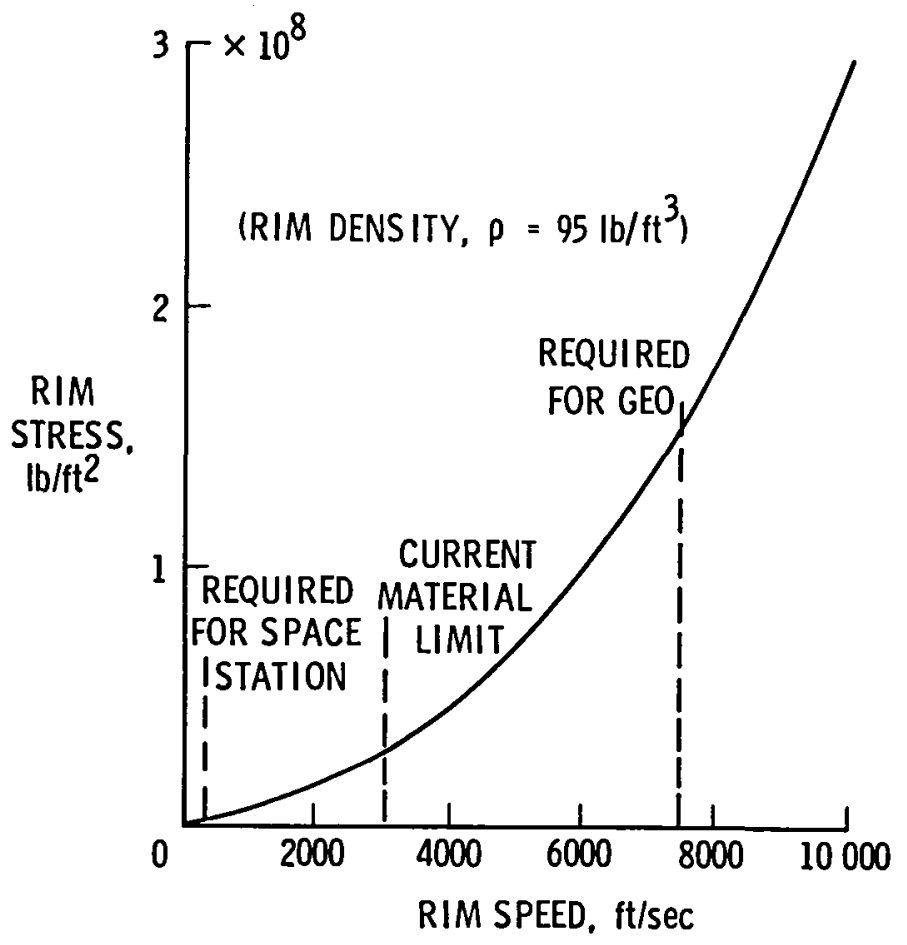

Fig. 6 Rim stress versus speed. 


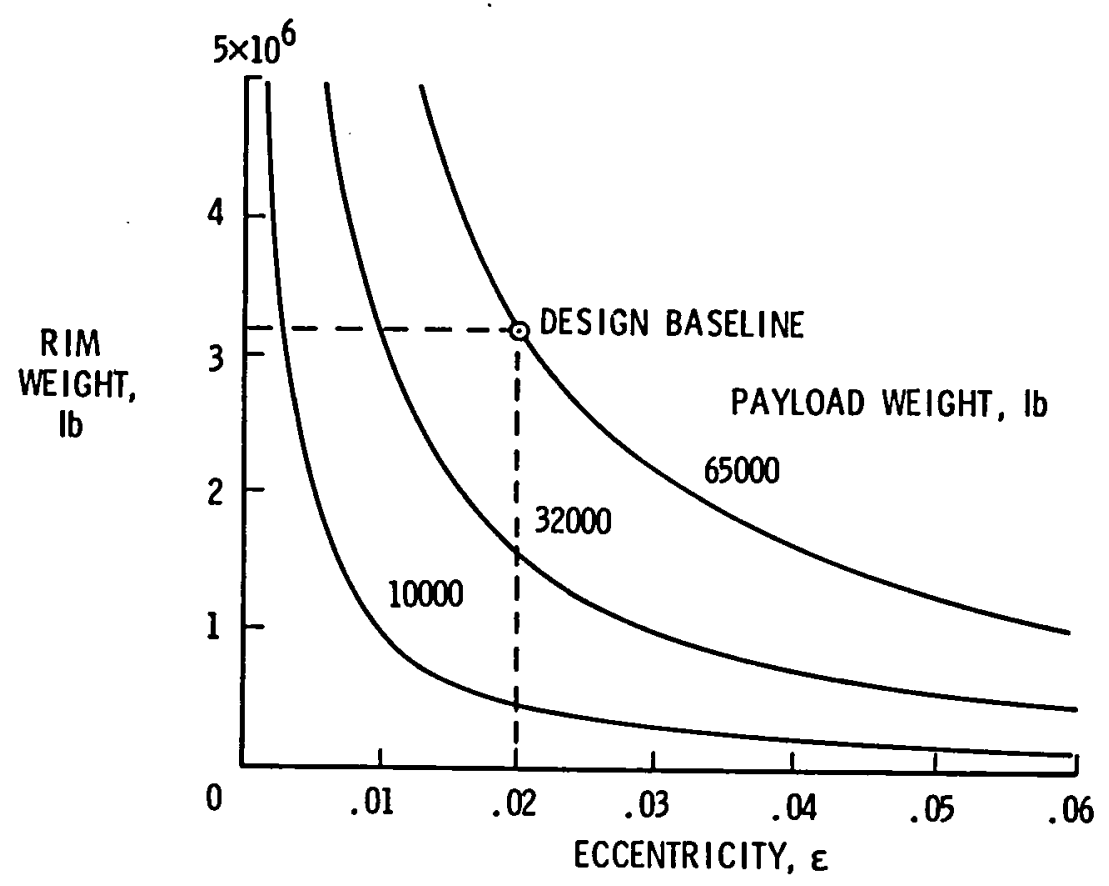

Fig. 7 Rim weight versus eccentricity for several payloads.

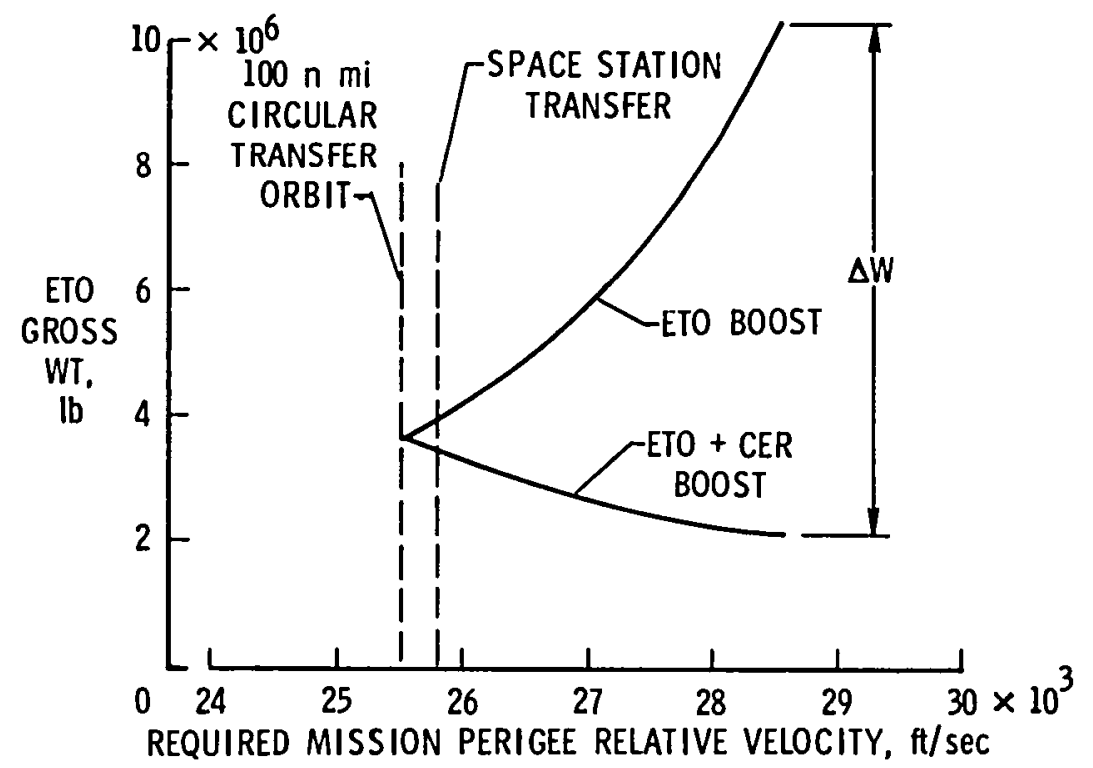

Fig. 8 Earth-to-orbit launch vehicle size versus perigee velocity with and without a Capture-Ejector Satellite. 


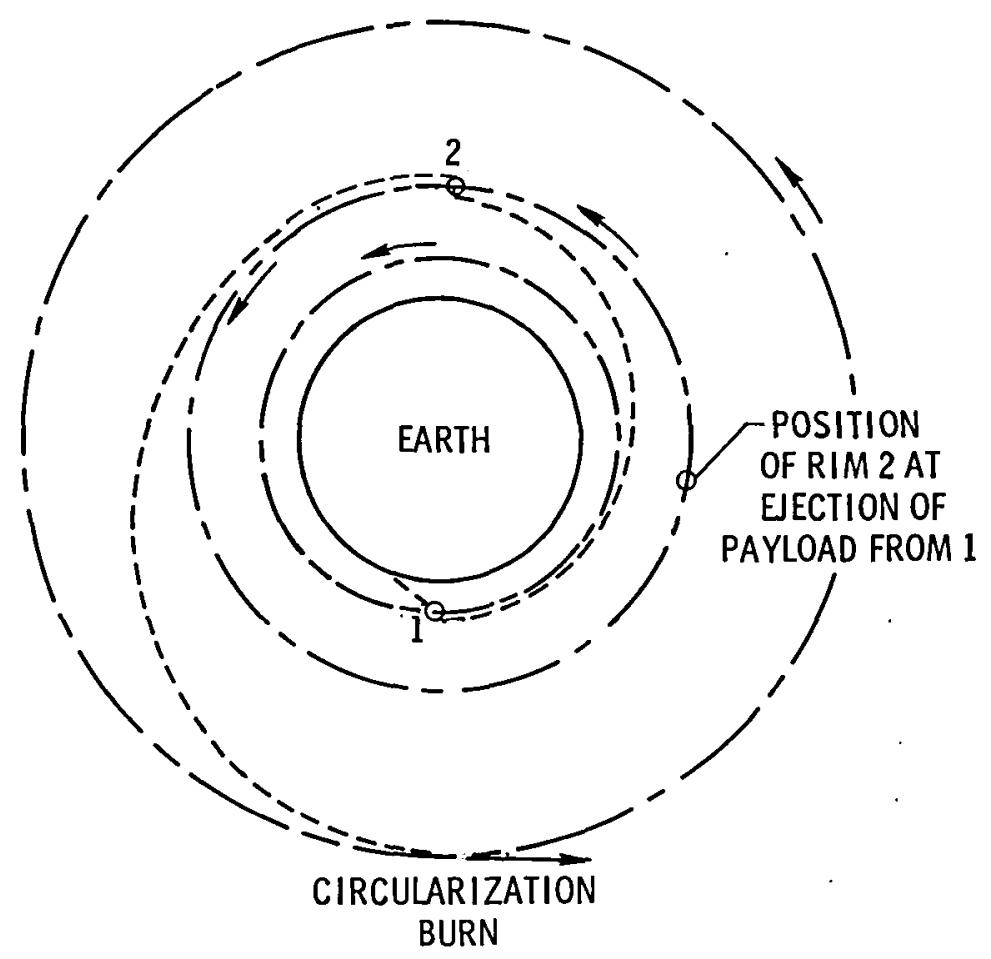

Fig. 9 Multiple Capture-Ejector Satellites for orbital transfer.

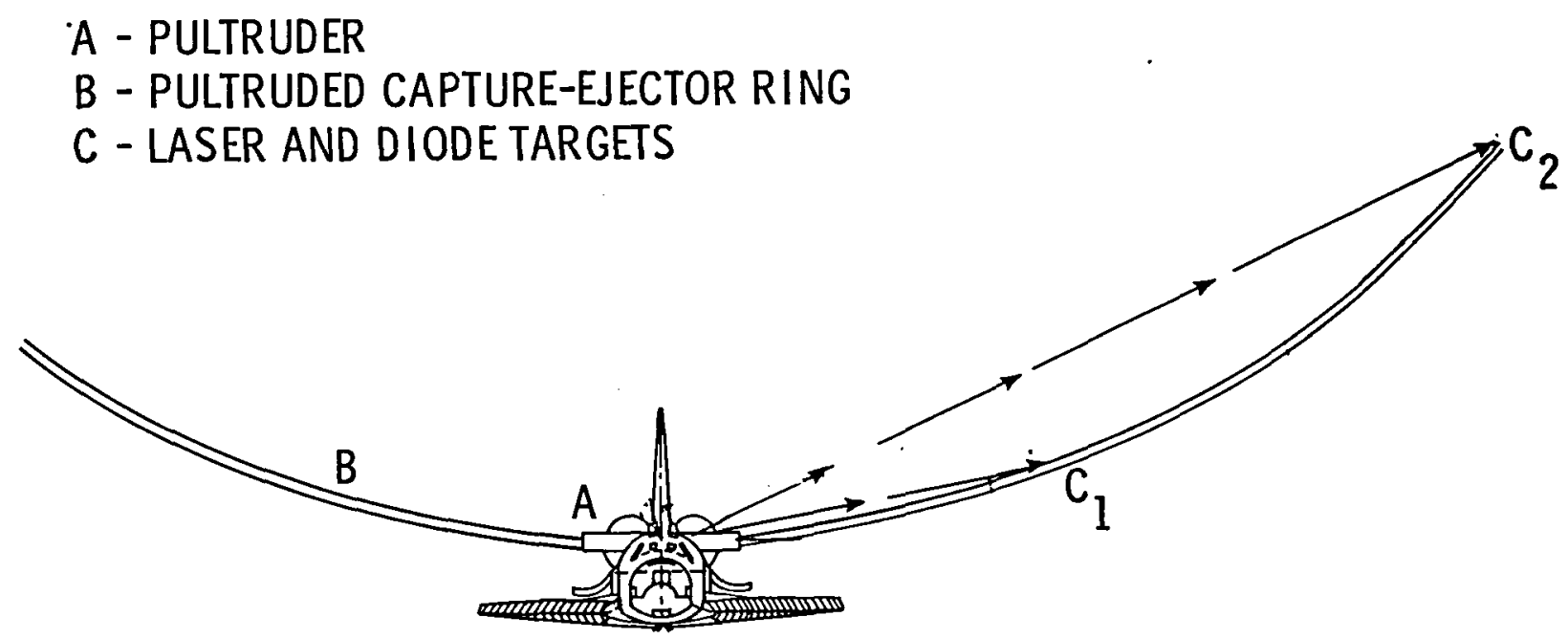

Fig. 10 Capture-Ejector Satellite manufacture. 


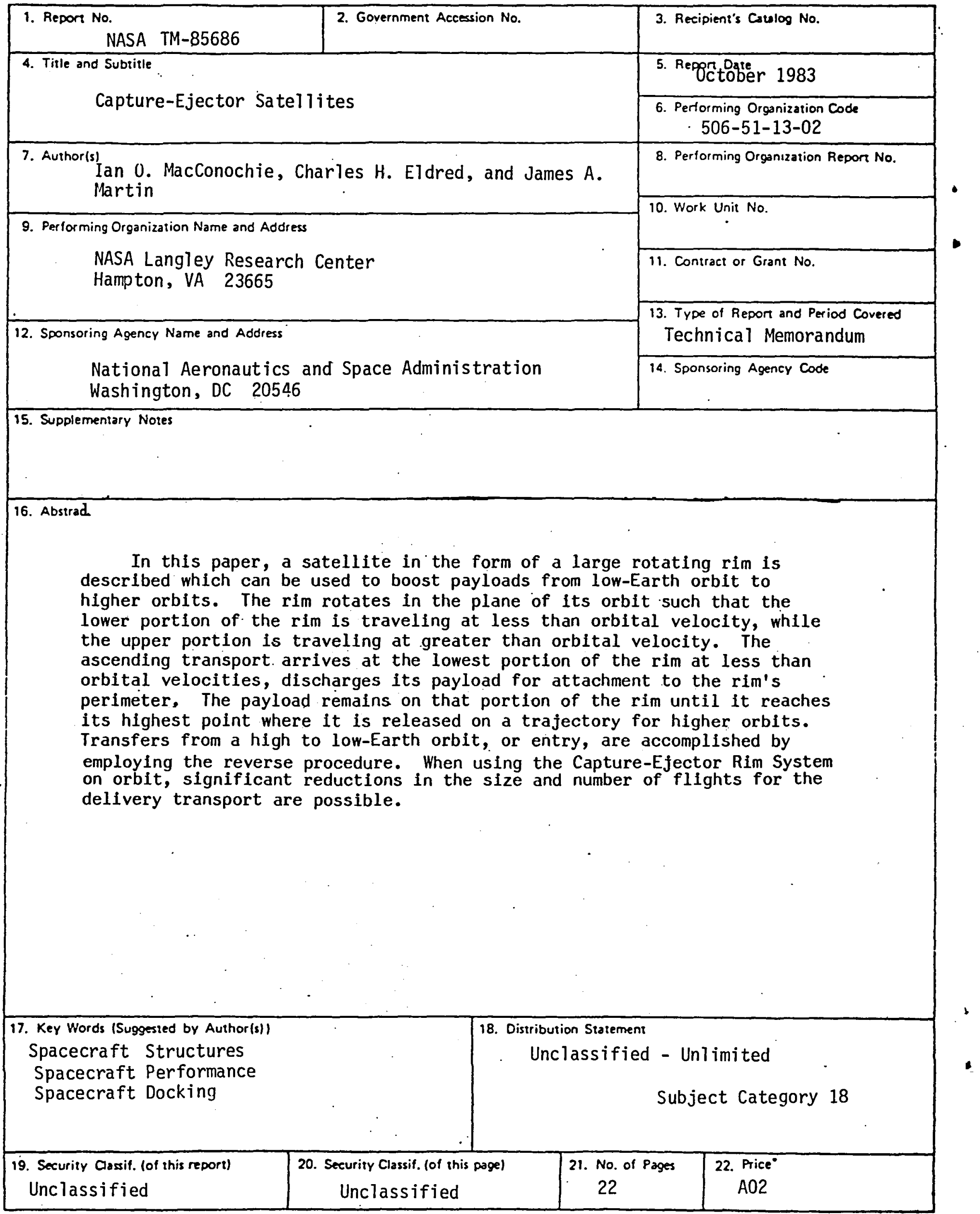

* For sale by the National Technical Information Service. Springlield. Virgina 22161 
